\title{
SPIRE spectroscopy of the prototypical Orion Bar photodissociation region ${ }^{\star}$
}

\author{
E. Habart ${ }^{1}$, E. Dartois ${ }^{1}$, A. Abergel ${ }^{1}$, J.-P. Baluteau ${ }^{2}$, D. Naylor ${ }^{13}$, E. Polehampton ${ }^{13,14}$, C. Joblin ${ }^{5,6}$, P. Ade ${ }^{3}$, \\ L. D. Anderson ${ }^{2}$, P. André ${ }^{4}$, H. Arab ${ }^{1}$, J.-P. Bernard ${ }^{6}$, K. Blagrave ${ }^{11}$, S. Bontemps ${ }^{18}$, F. Boulanger ${ }^{1}$, M. Cohen ${ }^{7}$, \\ M. Compiegne ${ }^{11}$, P. Cox ${ }^{8}$, G. Davis ${ }^{9}$, R. Emery ${ }^{14}$, T. Fulton ${ }^{17}$, C. Gry ${ }^{2}$, M. Huang ${ }^{10}$, S. C. Jones ${ }^{13}$, J. Kirk ${ }^{3}$, \\ G. Lagache ${ }^{1}$, T. Lim ${ }^{14}$, S. Madden ${ }^{4}$, G. Makiwa ${ }^{13}$, P. Martin ${ }^{11}$, M.-A. Miville-Deschênes ${ }^{1}$, S. Molinari ${ }^{12}$, \\ H. Moseley ${ }^{16}$, F. Motte ${ }^{4}$, K. Okumura ${ }^{4}$, D. Pinheiro Gonçalves ${ }^{11}$, J. Rodon ${ }^{2}$, D. Russeil ${ }^{2}$, P. Saraceno ${ }^{12}$, S. Sidher ${ }^{14}$, \\ L. Spencer ${ }^{13}$, B. Swinyard ${ }^{14}$, D. Ward-Thompson ${ }^{3}$, G. J. White ${ }^{14,15}$, and A. Zavagno ${ }^{2}$
}

(Affiliations are available in the online edition)

Received 31 March 2010 / Accepted 14 May 2010

\section{ABSTRACT}

Aims. We present observations of the Orion Bar photodissociation region (PDR) obtained with the SPIRE instrument on-board Herschel. Methods. We obtained SPIRE Fourier-transform spectrometer (FTS) sparse sampled maps of the Orion bar.

Results. The FTS wavelength coverage and sensitivity allow us to detect a wealth of rotational lines of CO (and its isotopologues), fine structure lines of $\mathrm{C}$ and $\mathrm{N}^{+}$, and emission lines from radicals and molecules such as $\mathrm{CH}^{+}, \mathrm{CH}, \mathrm{H}_{2} \mathrm{O}$ or $\mathrm{H}_{2} \mathrm{~S}$. For species detected from the ground, our estimates of the column densities agree with previously published values. The comparison between ${ }^{12} \mathrm{CO}$ and ${ }^{13} \mathrm{CO}$ maps shows particularly the effects of optical depth and excitation in the molecular cloud. The distribution of the ${ }^{12} \mathrm{CO}$ and ${ }^{13} \mathrm{CO}$ lines with upper energy levels indicates the presence of warm $(\sim 100-150 \mathrm{~K}) \mathrm{CO}$. This warm CO component is a significant fraction of the total molecular gas, confirming previous ground based studies.

Key words. infrared: ISM - ISM: lines and bands - ISM: molecules - evolution - submillimeter: ISM - ISM: general

\section{Introduction}

The Orion Bar located between the Orion molecular cloud and the HII region surrounding the Trapezium stars is one of the beststudied photodissociation regions (PDRs) in the Galaxy. Much of the emission from massive star-forming regions will originate from these interfaces, which are responsible for reprocessing the energy output from stars and reemitting this energy at infrared-millimetre wavelengths including a rich mixture of gas lines (i.e., Hollenbach \& Tielens 1999). Visible-ultraviolet stellar radiation governs the chemical and thermal state of the gas in these regions. The impinging radiation field on the Bar is $\chi=(0.5-2.5) \times 10^{4} \chi_{0}$ (Tielens \& Hollenbach 1985; Marconi et al. 1998), where $\chi_{0}$ is the Solar neighbourhood far-UV interstellar radiation field as given by Draine (1978). The UV field varies as a function of depth within the cloud, providing a unique opportunity to study how the dust populations and the molecular content evolve with the excitation and physical conditions. This is important for the evolution of the cloud and its associated star formation.

The ESA Herschel Space Observatory (Pilbratt et al. 2010) offers a unique opportunity to observe continuously between $\sim 55$ and $672 \mu \mathrm{m}$. This range includes most of the PDR gas lines and dust components emission and will provide a fundamental step in our understanding of the evolution of the interstellar matter. Here, we present a first analysis of Fourier-transform

* Herschel is an ESA space observatory with science instruments provided by European-led Principal Investigator consortia and with important participation from NASA. spectrometer (FTS) observations of the Orion Bar obtained with the SPIRE instrument (Griffin et al. 2010) on-board Herschel.

\section{Observations with the FTS}

The SPIRE FTS simultaneously measures the source spectrum across two wavebands: spectrometer long wavelength (SLW), covering 14.9-33.0 $\mathrm{cm}^{-1}(303-671 \mu \mathrm{m})$ and spectrometer short wavelength (SSW) covering 32.0-51.5 $\mathrm{cm}^{-1}(194-313 \mu \mathrm{m})$. Each band is imaged with a hexagonal bolometer array with pixel spacing of approximately twice the beam-width. The FWHM beam-widths of the SLW and SSW arrays vary between $29-42^{\prime \prime}$ and $17-21^{\prime \prime}$ respectively. The source spectrum, including the continuum, is obtained by taking the inverse transform of the observed interferogram. For more details on the FTS calibration and data reduction procedures, the reader is referred to the article by Swinyard et al. (2010).

Our observations are part of the "Evolution of Interstellar dust" key program of the SPIRE consortium (Abergel et al. 2010). The Orion Bar was observed with a single pointing in the high-resolution mode of the SPIRE FTS on 2009 September 13 (Herschel observation ID, 1342183819). Two scan repetitions were observed which gave an on-source integration time of 266.4 seconds. The pointing centre was at a right ascension and declination $(\mathrm{J} 2000)$ of $05^{\mathrm{h}} 35^{\mathrm{m}} 22.83^{\mathrm{s}}$ and $-05^{\circ} 24^{\prime} 57.67^{\prime \prime}$ (see Fig. 1). The unapodized spectral resolution was $0.04 \mathrm{~cm}^{-1}$ $(1.2 \mathrm{GHz})$. After apodization (using extended Norton-Beer function 1.5; Naylor \& Tahic 2007) the FWHM of the resulting instrument line shape is $0.0724 \mathrm{~cm}^{-1}(2.17 \mathrm{GHz})$. 

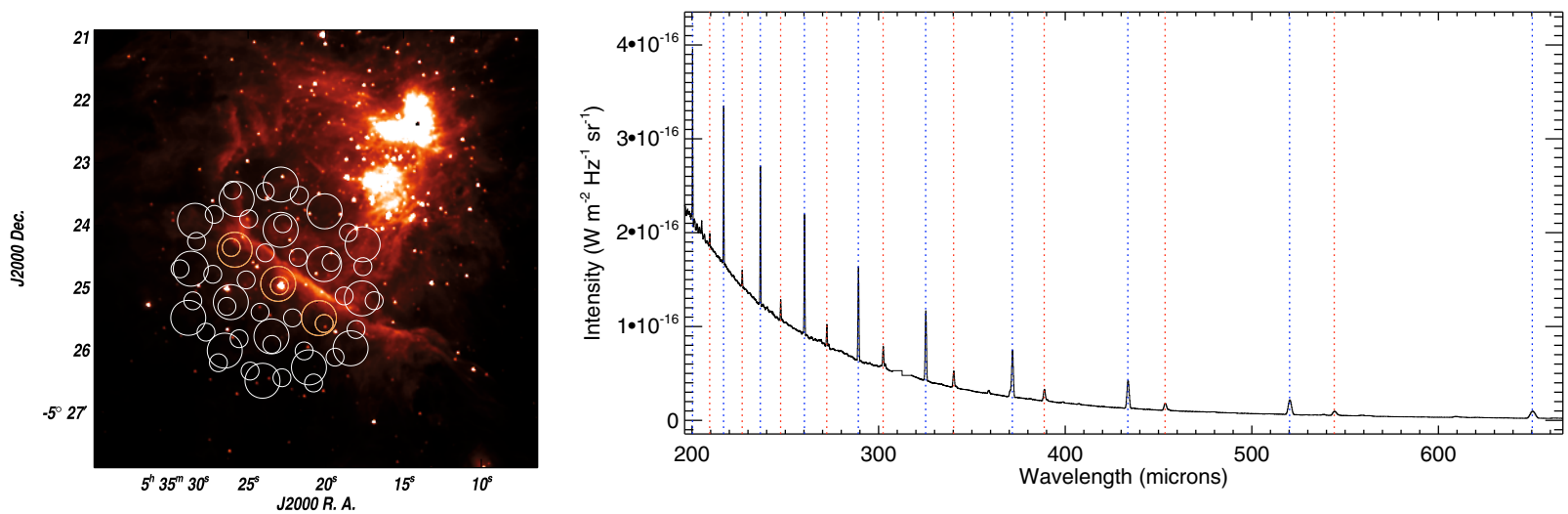

Fig. 1. Left: map of the Orion Bar obtained with Spitzer (IRAC at $3.8 \mu \mathrm{m}$ ) with the SPIRE SLW (large circle) and SSW (small circle) array positions marked. The PDR is wrapped around the HII region created by the Trapezium stars (right corner) and changes from a face-on to an edgeon geometry where the emission peaks. Right: averaged apodized FTS spectra over the three arrays on the Bar (yellow large and small circles). The blue and red dotted lines delinate the ${ }^{12} \mathrm{CO}$ and ${ }^{13} \mathrm{CO}$ lines position respectively.

While unapodized FTS spectra provide the highest spectral resolution, the instrument line shape, which for an ideal FTS is the classical sinc function, is characterized by relatively large secondary oscillations with negative lobes. An iterative spectralline fitting routine was developed to extract line parameters from unapodized FTS spectra (Jones et al. 2009). This algorithm fits a continuum (either a low order polynomial or a blackbody variant) and a series of lines with the Levenberg-Marquardt leastsquares method. The fitting procedure weights the spectral intensity at a given frequency of an averaged spectrum by the statistical uncertainty at that frequency. The fitting routine returns the line centres, intensities, and line widths, together with their associated errors.

\section{Results}

\subsection{Detected gas lines}

The averaged apodized FTS spectra over the three SLW/SSW detectors aligned on the Bar and corrected for obliquity effects ${ }^{1}$ are presented in Figs. 1 and 2. The FTS wavelength coverage allows us to detect a wealth of rotational lines of $\mathrm{CO}$ (and its isotopologues), fine structure lines of $\mathrm{C}$ and $\mathrm{N}^{+}$, and emission lines from several radicals and molecules. The expected line positions for detected species are marked in Figs. 1 and 2. The ${ }^{12} \mathrm{CO}$ transitions, which appear as the bright narrow lines, are here seen for the first time together from $J=4-3$ to $13-12$ in a single spectrum. The ${ }^{13} \mathrm{CO}$ lines are clearly detected from $J=5-4$ to 13-12. Most of the $\mathrm{C}^{18} \mathrm{O}$ lines are visible but blended with the ${ }^{13} \mathrm{CO}$ lines; some $\mathrm{C}^{17} \mathrm{O}$ lines are detected. One emission line at about $359 \mu \mathrm{m}$ lies at the position of the fundamental rotational transition of $\mathrm{CH}^{+}$(Naylor et al. 2010). This detection can be related to the observation of the $\mathrm{CH}$ lambda doublet transitions at about $556.5 \mu \mathrm{m}$ and $560.7 \mu \mathrm{m}$, although it is possibly blended with an $\mathrm{HCO}^{+} J=6 \rightarrow 5$ line. The ortho- $\mathrm{H}_{2} \mathrm{O} 1_{10} \rightarrow 1_{01}$ line at $\sim 538$ and para- $\mathrm{H}_{2} 2_{11} \rightarrow 2_{02}$ at $398 \mu \mathrm{m}$ are clearly detected. The $\sim 269 \mu \mathrm{m}$ para- $\mathrm{H}_{2} \mathrm{O} 1_{11} \rightarrow 0_{00}$ line was also detected, but the signal-to-noise ratio is low. Some other $\mathrm{H}_{2} \mathrm{O}$ lines may be blended. The $\mathrm{H}_{2} \mathrm{~S} 2_{12} \rightarrow 1_{01}$ line at $\sim 407 \mu \mathrm{m}$ is detected, while other fainter $\mathrm{H}_{2} \mathrm{~S}$ lines at shorter wavelengths are only marginally detected. Some features related to the emission of $\mathrm{HCO}^{+}, \mathrm{HCN}, \mathrm{CN}$ and $\mathrm{C}_{2} \mathrm{H}$ are observed as expected (e.g., Hogerheijde et al. 1995; Simon et al. 1997; Young Owl et al. 2000; Teyssier et al. 2004; van Der Wiel et al. 2009), but to help

\footnotetext{
1 The obliquity effect is important at the highest frequencies, where a significant error in the line position is introduced.
}
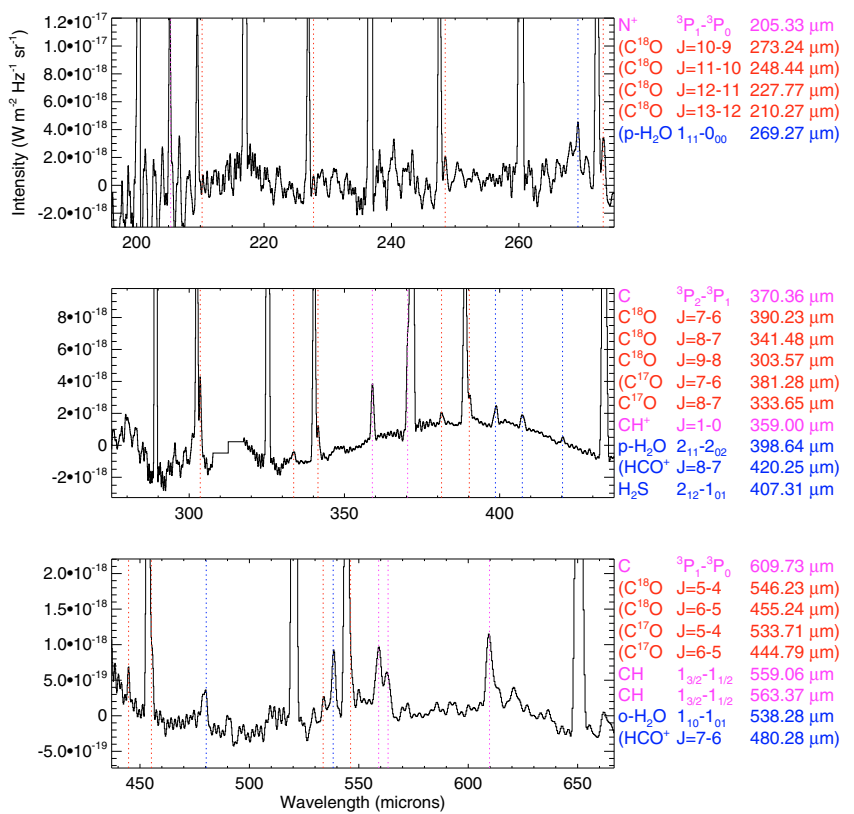

Fig. 2. Zoom of the averaged apodized FTS spectra continuum substracted. Dotted lines show the positions where specific gas lines are expected, excluding the ${ }^{12} \mathrm{CO}$ and ${ }^{13} \mathrm{CO}$ lines shown in Fig. 1. The corresponding lines and wavelengths are marked on the right. Lines between brackets are only possibly detected at this level of analysis.

distinguish the spectral confusion for fainter lines or unresolved k-ladder transitions from species such as methanol, the actual signal-to-noise ratio will be improved as the SPIRE FTS response is better understood and, scheduled deeper observations will also help.

\subsection{Mapping gas lines}

Figure 3 presents sparse sampled ${ }^{2}$ maps of nearly the complete $\mathrm{CO}$ and ${ }^{13} \mathrm{CO}$ band measured. Off-axis calibrations are not guaranteed because both detector arrays have not yet been fully characterised. The comparison between these maps shows the effects of optical depth and excitation in the molecular cloud particularly well. The emission of the less abundant ${ }^{13} \mathrm{CO}$ isotopologue

2 The present FTS science demonstration phase observations sparsely samples the field of view and do not allow us to present fully sampled maps. 


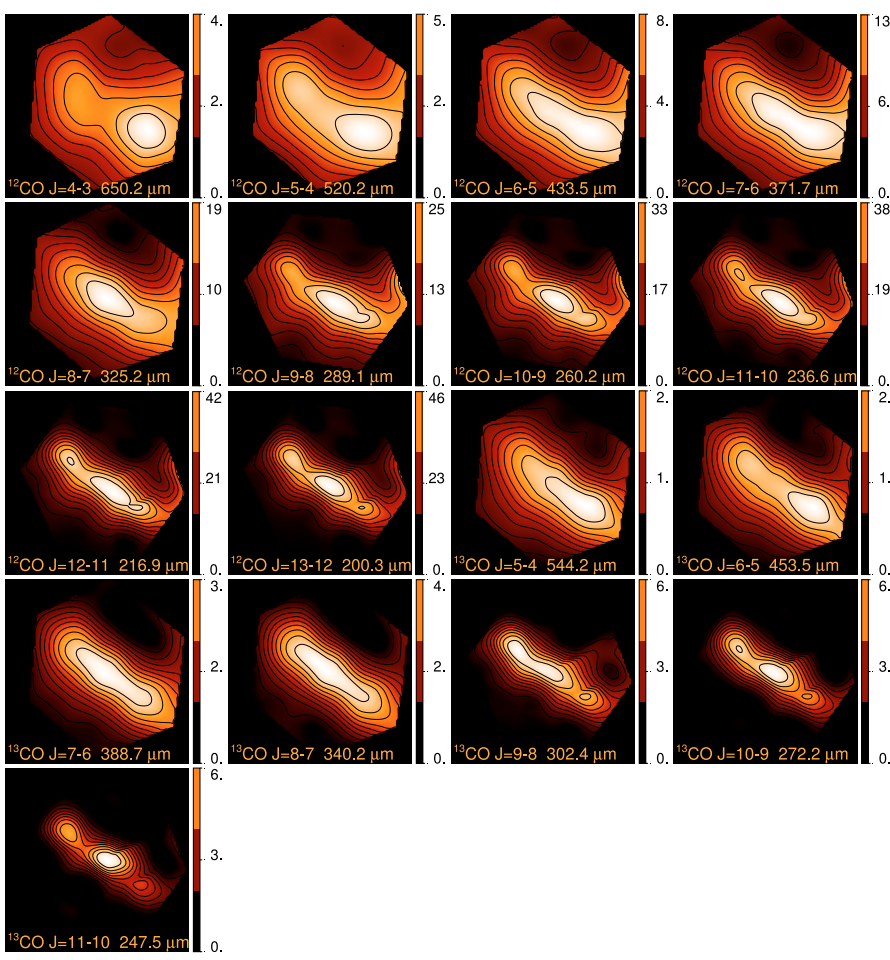

Fig. 3. Sparse sampled maps in the ${ }^{12} \mathrm{CO}$ and ${ }^{13} \mathrm{CO}$ lines measured, except for the ${ }^{13} \mathrm{CO} J=12-11$ at $\sim 227$ and $J=13-12$ at $209 \mu \mathrm{m}$ lines. Scales are in $10^{5} \mathrm{erg} \mathrm{s}^{-1} \mathrm{~cm}^{-2} \mathrm{sr}^{-1}$. Contour levels are drawn at $10,20, \ldots$, $90 \%$ of the maximum intensity.

probes the denser shielded regions, while the ${ }^{12} \mathrm{CO}$ optically thick emission likely comes from the less dense surface layers (Lis et al. 1998). The highest rotational lines, which are very sensitive to both gas densities and temperatures, show strong and peaked emission on the Bar, while they are not visible in the off Bar positions. Emission lines of species such as $\mathrm{C}, \mathrm{N}^{+}$or $\mathrm{CH}^{+}$ show spatially extended emission.

\subsection{Molecular column densities}

We used the observed line intensities and the CASSIS software ${ }^{3}$ to estimate the beam-averaged molecular column densities. We list in Table 1 column densities estimated for a volume density of $10^{5} \mathrm{~cm}^{-3}$ as applicable to the extended molecular gas in the Bar (Hogerheijde et al. 1995) and in the high-density limit, because some of the line emission may originate from dense clumps $^{4}$. We adopt the mean molecular gas temperature towards the Bar of $\sim 85 \mathrm{~K}$ for kinetic temperatures as determined from the ground Hogerheijde et al. (1995), and the more extreme 50 to $150 \mathrm{~K}$ range values that probe different zones of the PDR (Lis \& Schilke 2003; Batrla \& Wilson 2003). The line widths were taken equal to $3 \mathrm{~km} \mathrm{~s}^{-1}$, following previous higher resolution observations (Hogerheijde et al. 1995; Johnstone et al. 2003).

3 Based on analysis carried out with the CASSIS software and CDMS, JPL spectroscopic databases and RADEX (van Der Tak et al. 2007) molecular databases. CASSIS has been developed by CESRUPS/CNRS (http://cassis.cesr.fr).

4 The clumpiness of the PDR inferred by Hogerheijde et al. (1995) was confirmed by interferometric data of Young Owl et al. (2000); Lis \& Schilke (2003). Clump densities up to $10^{7} \mathrm{~cm}^{-3}$ were derived by Lis \& Schilke (2003), while the density of the interclump medium should fall between a few $10^{4} \mathrm{~cm}^{-3}$ (Young Owl et al. 2000) and $2 \times 10^{5} \mathrm{~cm}^{-3}$ (Simon et al. 1997).
Our values for the column densities agree for species detected from the ground with previously published values to within a factor of 2-3: Hogerheijde et al. (1995) for $\mathrm{C}^{18} \mathrm{O}$ and $\mathrm{HCO}^{+}$; Johnstone et al. (2003) for $\mathrm{C}^{17} \mathrm{O}$; and Leurini et al. (2006) for $\mathrm{H}_{2} \mathrm{~S}$. Beam dilution effects could introduce a significant factor. To convert the observed $\mathrm{C}^{18} \mathrm{O} J=8-7$ and $\mathrm{C}^{17} \mathrm{O} \mathrm{J}=8-7$ line intensities to a total $\mathrm{H}_{2}$ column density, we assume isotopic ratios ${ }^{16} \mathrm{O} /{ }^{18} \mathrm{O} \sim 560,{ }^{16} \mathrm{O} /{ }^{17} \mathrm{O} \sim 1800$ (Wilson \& Rood 1994) and a relative $\mathrm{CO}$ abundance to $\mathrm{H}_{2}$ of $1.1 \times 10^{-4}$ as applicable for the Orion Bar PDR (Johnstone et al. 2003). We find $N\left(\mathrm{H}_{2}\right) \sim 9 \times 10^{22} \mathrm{~cm}^{-2}$ assuming $T \sim$ $85 \mathrm{~K}$, which implies the following molecular abundances on the Bar: $x\left(\right.$ ortho- $\left.\mathrm{H}_{2} \mathrm{O}\right) \leq 3.3_{-1.7}^{+3.3} \times 10^{-7} ; x\left(\right.$ para- $\left.\mathrm{H}_{2} \mathrm{O}\right) \leq 5_{-3.1}^{+11.7} \times$ $10^{-7} ; x\left(\mathrm{HCO}^{+}\right) \leq 3.9_{-2.1}^{+8.3} \times 10^{-9} ; x\left(\mathrm{CH}^{+}\right)=7.2_{-0.7}^{+2.6} \times 10^{-11}$; $x\left(\mathrm{H}_{2} \mathrm{~S}\right)=3.4_{-1}^{+2.3} \times 10^{-10}$.

$\mathrm{H}_{2} \mathrm{O}$ is extremely sensitive to the local physical conditions in molecular clouds: close to the surface, molecules are photodissociated, while deeper into the cloud molecules freeze onto grain surfaces (i.e., Hollenbach et al. 2009). Desorption of ices (Westley et al. 1995; Seperuelo Duarte et al. 2009) could supply gas-phase species. The high abundances of sulphur species remain an interesting puzzle for interstellar chemistry (i.e., Goicoechea et al. 2006). The observed abundance of species such as $\mathrm{H}_{2} \mathrm{~S}$ are difficult to interpret in models. $\mathrm{H}_{2} \mathrm{~S}$ results from a mixed chemistry involving gas-phase reactions and grain-related processes.

\subsection{CO excitation}

Figure 4 shows the distribution of the ${ }^{12} \mathrm{CO}$ and ${ }^{13} \mathrm{CO}$ line intensities as a function of the upper energy levels. The observed intensities of the optically thick ${ }^{12} \mathrm{CO}$ lines provide an estimate of the temperature of about $\sim 85 \mathrm{~K}$ across the bar, consistent with many observed transitions from the ground. However, the ${ }^{12} \mathrm{CO}$ $J=12-11$ and $J=13-12$ transitions do not agree with that temperature (intensities higher by a factor of 2 and 4 respectively), which is consistent with ground data of the $J=14-13$ transition dominated by warmer CO (Stacey et al. 1993; Tauber et al. 1994). Similarly, the distribution of the ${ }^{13} \mathrm{CO}$ lines cannot be described by a single temperature. A dense and warm $(\sim 100-$ $150 \mathrm{~K}$ ) component with a significant column density (about $15 \%$ of the total column) is required to fit the observed line intensities with $J_{u} \geq 9$. We find that the ${ }^{13} \mathrm{CO}$ lines become optically thin for $J_{u} \geq 9$, while all the ${ }^{12} \mathrm{CO}$ lines are optically thick.

We computed PDR models for the Bar with an updated version of the Meudon PDR code described in Le Petit et al. (2006) solving in an iterative way the chemical and thermal balances at each point of the cloud. Adopting $\chi=10^{4} \chi_{0}$, a constant gas density of $10^{5} \mathrm{~cm}^{-3}$ or constant thermal gas pressure of $8 \times 10^{7} \mathrm{~K} \mathrm{~cm}^{-3}$ (Allers et al. 2005), the models predict a gas temperature of about $\sim 50-80 \mathrm{~K}$ for the $\mathrm{CO}$ emitting gas. Consequently, it cannot explain the observed warm CO. One explanation is that the warm CO originates from dense clumps at the PDR surface (Tauber et al. 1994). An alternative solution are additional heating mechanisms for the interior, like shocks (or turbulence) or cosmic ray heating (Pellegrini et al. 2009). Outof-equilibrium effects such as advection of molecular gas from the shielded cloud interior to the warm surface could also enhance the column densities of warm CO. Progress is expected from the spectroscopy of additional cooling lines, to be obtained from Herschel PACS and HIFI instruments. In particular, observations of gas cooling lines at high spectral resolution with HIFI will provide missing information about the gas velocity within 
Table 1. Beam-averaged molecular column densities.

\begin{tabular}{llllll}
\hline \hline Species & Transition & $\begin{array}{l}\text { Wavelength } \\
\text { (microns) }\end{array}$ & $\begin{array}{l}E_{u} \\
(\mathrm{~K})\end{array}$ & $\begin{array}{l}\text { Intensity } \\
\left(10^{-6} \mathrm{erg} \mathrm{s}^{-1} \mathrm{~cm}^{-2} \mathrm{sr}^{-1}\right)\end{array}$ & $\begin{array}{l}\text { Column density } \\
\left(10^{15} \mathrm{~cm}^{-2}\right)\end{array}$ \\
\hline $\mathrm{C}^{18} \mathrm{O}$ & $J=7 \rightarrow 6$ & 390.2 & 147.5 & $2.6[1.1]$ & $7.7[24,3.8]^{b}$ \\
$\mathrm{C}^{18} \mathrm{O}$ & $J=8 \rightarrow 7$ & 341.5 & 189.6 & $3.3[1.1]$ & $15[72,5.5]^{b}$ \\
$\mathrm{C}^{18} \mathrm{O}$ & $J=9 \rightarrow 8$ & 303.6 & 237.0 & $11[3.9]$ & $96[720,25]^{b}-10[43,5.4]^{c}$ \\
\hline $\mathrm{C}^{17} \mathrm{O}$ & $J=8 \rightarrow 7$ & 333.7 & 194.1 & $1.4[1.1]$ & $6.6[33,2.3]^{b}$ \\
\hline $\mathrm{CH}^{+}$ & $J=1 \rightarrow 0$ & 359.0 & 40.1 & $6.7[1.1]$ & $0.0065[0.0058,0.0088]^{c}$ \\
\hline ortho- $\mathrm{H}_{2} \mathrm{O}$ & $1_{10} \rightarrow 1_{01}$ & 538.3 & 61.0 & $2.6[1.1]$ & $\leq 30[60,15]^{b, d}$ \\
para- $\mathrm{H}_{2} \mathrm{O}$ & $2_{11} \rightarrow 2_{02}$ & 398.6 & 136.9 & $2.0[1.1]$ & $\leq 45[150,17]^{b, d}$ \\
\hline $\mathrm{HCO}^{+}$ & $J=8 \rightarrow 7$ & 420.3 & 154.1 & $\leq 0.7^{e}$ & $\leq 0.35[1.1,0.16]^{b, e}$ \\
\hline $\mathrm{H}_{2} \mathrm{~S}$ & $2_{12} \rightarrow 1_{01}$ & 407.3 & 55.1 & $1.4[1.1]$ & $0.031[0.023,0.052]^{c}$ \\
\hline
\end{tabular}

Notes. ${ }^{(a)}$ Intensities with uncertainty in between brackets. ${ }^{(b)}$ Beam-averaged column densities using RADEX with $n=10^{5} \mathrm{~cm}^{-3}$ and $T=85 \mathrm{~K}$, with estimations for 50 and $150 \mathrm{~K}$ in between brackets. ${ }^{(c)}$ Beam-averaged column densities in the high-density (LTE) limit for $85 \mathrm{~K}$, with estimations for 50 and $150 \mathrm{~K}$ in between brackets. ${ }^{(d)}$ Note that the density (or temperature) could be higher than assumed, which would decrease the column density, and the para- $\mathrm{H}_{2} \mathrm{O} 2_{11} \rightarrow 2_{02}$ line could be affected by IR pumping. ${ }^{(e)}$ We used the $\mathrm{HCO}^{+} J=8 \rightarrow 7$ line because the $\mathrm{HCO}^{+}$ $J=7 \rightarrow 6$ line is possibly blended with an $\mathrm{HCl} J=1 \rightarrow 0$ line. Column densities from ${ }^{12} \mathrm{CO}$ and ${ }^{13} \mathrm{CO}$ lines are uncertain due to high optical depth and are not listed.

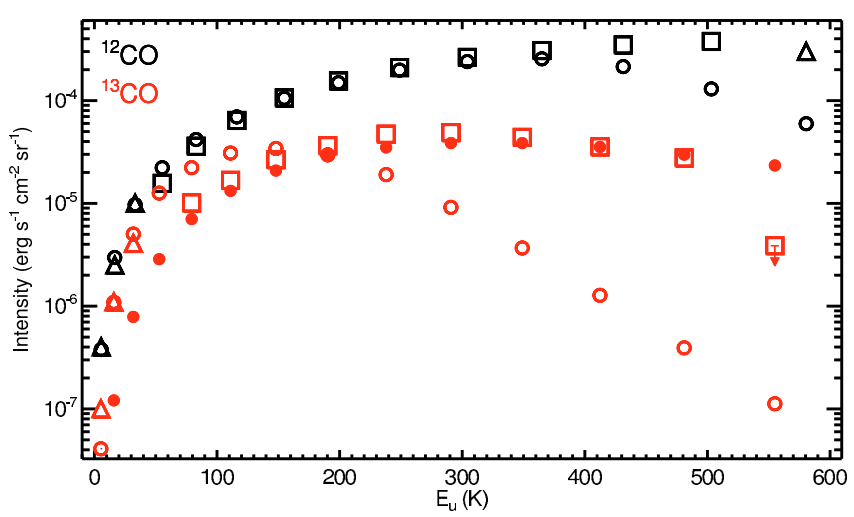

Fig. 4. Distribution of the ${ }^{12} \mathrm{CO}$ (black) and ${ }^{13} \mathrm{CO}$ (red) line intensities as a function of the upper energy levels. The squares show the FTS data complemented by ground-based measurements shown by triangles (Stacey et al. 1993; Tauber et al. 1994; White \& Sandell 1995; Hogerheijde et al. 1995; van Der Wiel et al. 2009). Error bars are small compared to the symbol size. The arrows indicate upper limits. The empty circles show the RADEX calculation for $T=85 \mathrm{~K}$, $n=10^{5} \mathrm{~cm}^{-3}, N\left({ }^{12} \mathrm{CO}\right)=10^{19} \mathrm{~cm}^{-2}$ and $N\left({ }^{13} \mathrm{CO}\right)=1.3 \times 10^{17} \mathrm{~cm}^{-2}$. The full circles show the LTE calculation for $T=120 \mathrm{~K}$ and $\mathrm{N}\left({ }^{13} \mathrm{CO}\right)=2 \times 10^{16} \mathrm{~cm}^{-2}$.

the PDR and allow us to assign some lines that could be merged in the lower resolution SPIRE spectra.

\section{Conclusions}

We have analysed the first spectral survey taken in the Orion Bar by the FTS of SPIRE. A wealth of rotational lines of CO (and its isotopologues), fine structure lines of $\mathrm{C}$ and $\mathrm{N}^{+}$, and emission lines from radicals and molecules were found. We present the first sparse sampled maps, which illustrate FTS line mapping capabilities. We discussed the $\mathrm{CO}$ excitation and emphasized the need for complementary spectroscopic data.

Acknowledgements. We are grateful to J. R. Goicoechea and M. Gerin for relevant comments and suggestions. SPIRE has been developed by a consortium of institutes led by Cardiff Univ. (UK) and including Univ. Lethbridge (Canada); NAOC (China); CEA, LAM (France); IFSI, Univ. Padua (Italy); IAC (Spain); Stockholm Observatory (Sweden); Imperial College London, RAL,
UCL-MSSL, UKATC, Univ. Sussex (UK); Caltech, JPL, NHSC, Univ. Colorado (USA). This development has been supported by national funding agencies: CSA (Canada); NAOC (China); CEA, CNES, CNRS (France); ASI (Italy); MCINN (Spain); SNSB (Sweden); STFC (UK); and NASA (USA).

\section{References}

Abergel, A., Arab, H., Compiègne, M., et al. 2010, A\&A, 518, L96

Allers, K. N., Jaffe, D. T., Lacy, J. H., Draine, B. T., \& Richter, M. J. 2005, ApJ, 630, 368

Batrla, W., \& Wilson, T. L. 2003, A\&A, 408, 231

Draine, B. T. 1978, ApJS, 36, 595

Goicoechea, J. R., Pety, J., Gerin, M., et al. 2006, A\&A, 456, 565

Griffin, M. J., Abergel, A., Abreu, A, et al. 2010, A\&A, 518, L3

Hogerheijde, M. R., Jansen, D. J., \& Van Dishoeck, E. F. 1995, A\&A, 294, 792

Hollenbach, D. J., \& Tielens, A. G. G. M. 1999, Rev. Modern Phys., 71, 173

Hollenbach, D., Kaufman, M. J., Bergin, E. A., \& Melnick, G. J. 2009, ApJ, 690, 1497

Johnstone, D., Boonman, A. M. S., \& van Dishoeck, E. F. 2003, A\&A, 412, 157

Jones, S., Naylor, D., Gom, B., \& Spencer, L. 2009, Proc. 30th Canadian Symposium on Remote Sensing

Le Petit, F., Nehmé, C., Le Bourlot, J., \& Roueff, E. 2006, ApJS, 164, 506

Leurini, S., Rolffs, R., Thorwirth, S., et al. 2006, A\&A, 454, L47

Lis, D. C., \& Schilke, P. 2003, ApJ, 597, L145

Lis, D. C., Serabyn, E., Keene, J., et al. 1998, ApJ, 509, 299

Marconi, A., Testi, L., Natta, A., \& Walmsley, C. M. 1998, A\&A, 330, 696

Naylor, D., \& Tahic, M. 2007, J. Opt. Soc. Am. A, 24, 3644

Naylor, D., Dartois, E., Habart, E., et al. 2010, A\&A, 518, L117

Pellegrini, E. W., Baldwin, J. A., Ferland, G. J., Shaw, G., \& Heathcote, S. 2009, ApJ, 693, 285

Pety, J., Teyssier, D., Fossé, D., et al. 2005, A\&A, 435, 885

Pilbratt, G. J., Riedinger, J. R., Passvogel, T., et al. 2010, A\&A, 518, L1

Seperuelo Duarte, E., Boduch, P., Rothard, H., et al. 2009, A\&A, 502, 599

Simon, R., Stutzki, J., Sternberg, A., \& Winnewisser, G. 1997, A\&A, 327, L9

Stacey, G. J., Jaffe, D. T., Geis, N., et al. 1993, ApJ, 404, 219

Swinyard, B. M., Ade, P., Baluteau, J.-P., et al. 2010, A\&A, 518, L4

Tauber, J. A., Tielens, A. G. G. M., Meixner, M., \& Foldsmith, P. F. 1994, ApJ, 422,136

Teyssier, D., Fossé, D., Gerin, M., et al. 2004, A\&A, 417, 135

Tielens, A. G. G. M., \& Hollenbach, D. 1985, ApJ, 291, 722

van Der Tak, F. F. S., Black, J. H., Schoeier, F. L., Jansen, D. J., \& van Dishoeck, E. F. 2007, A\&A, 468, 627

van Der Wiel, M. H. D., van Der Tak, F. F. S., Ossenkopf, V., et al. 2009, A\&A, 498, 161

Westley, M. S., Baragiola, R. A., Johnson, R. E., \& Baratta, G. A. 1995, Nature, 373, 405

White, G. J., \& Sandell, G. 1995, A\&A, 299, 179

Wilson, T. L., \& Rood, R. 1994, ARA\&A, 32, 191

Young Owl, R. C., Meixner, M. M., Wolfire, M., Tielens, A. G. G. M., \& Tauber, J. 2000, ApJ, 540, 886 
1 Institut d'Astrophysique Spatiale, UMR 8617, CNRS/Université Paris-Sud 11, 91405 Orsay, France

e-mail: emilie.habart@ias.u-psud.fr

2 Laboratoire d'Astrophysique de Marseille (UMR 6110 CNRS \& Université de Provence), 38 rue F. Joliot-Curie, 13388 Marseille Cedex 13, France

3 Department of Physics and Astronomy, Cardiff University, Cardiff, UK

${ }^{4}$ CEA, Laboratoire AIM, Irfu/SAp, Orme des Merisiers, 91191 Gif-sur-Yvette, France

5 CESR, Université de Toulouse, UPS, CESR, 9 Av. du colonel Roche, 31028 Toulouse Cedex 4, France

${ }^{6}$ CNRS, UMR5187, 31028 Toulouse, France

7 University of California, Radio Astronomy Laboratory, Berkeley, 601 Campbell Hall, US Berkeley CA 94720-3411, USA

8 Institut de Radioastronomie Millimétrique (IRAM), 300 rue de la Piscine, 38406 Saint-Martin-d'Hères, France
9 Joint Astronomy Centre, University Park, Hilo, USA

${ }_{10}$ National Astronomical Observatories, PR China

11 Canadian Institute for Theoretical Astrophysics, Toronto, Ontario, M5S 3H8, Canada

12 Istituto di Fisica dello Spazio Interplanetario, INAF, via del Fosso del Cavaliere 100, 00133 Roma, Italy

13 Institute for Space Imaging Science, University of Lethbridge, Lethbridge, Canada

14 The Rutherford Appleton Laboratory, Chilton, Didcot OX11 0QX, UK

15 Department of Physics \& Astronomy, The Open University, Milton Keynes MK7 6AA, UK

16 NASA - Goddard SFC, USA

17 Blue Sky Spectrosocpy Inc, Lethbridge, Canada

18 CNRS/INSU, Laboratoire d'Astrophysique 